\title{
Gestational Diabetes Mellitus and Risk of Childhood Overweight and Obesity in Offspring: A Systematic Review
}

\author{
Shin Y. Kim, J. Lucinda England, J. Andrea Sharma, and Terry Njoroge \\ Division of Reproductive Health, National Center for Chronic Disease Prevention and Health Promotion, \\ Centers for Disease Control and Prevention, Atlanta, GA 30341, USA \\ Correspondence should be addressed to Shin Y. Kim, skim1@cdc.gov
}

Received 1 June 2011; Accepted 22 July 2011

Academic Editor: Barbara Alexander

Copyright ( 2011 Shin Y. Kim et al. This is an open access article distributed under the Creative Commons Attribution License, which permits unrestricted use, distribution, and reproduction in any medium, provided the original work is properly cited.

We systematically reviewed research examining the association between gestational diabetes (GDM) and childhood overweight and obesity. We identified studies from three sources: (1) a PubMed search of articles published between January 1990-January 2011, (2) reference lists of publications from the PubMed search, and (3) reference lists of review articles. We included studies that examined GDM separately from pregestational diabetes and childhood overweight or obesity defined as BMI $>$ 85th or 95th percentile. A total of 12 studies were included in the systematic review. Crude odds ratios for the relationship between GDM and childhood overweight or obesity ranged from 0.7 to 6.3 ; in 8 studies, the associations were not statistically significant. In only 3 studies were results adjusted for any confounders; in the 2 that adjusted for prepregnancy obesity, the GDM and childhood overweight or obesity associations were attenuated and not statistically significant after adjustment. This paper demonstrates inconsistent evidence of an association between GDM and offspring overweight and obesity due to the methodological limitations of existing studies. Recommendations for future research are presented, which address methodological challenges.

\section{Introduction}

Approximately $4-6 \%$ of pregnancies in the United States are complicated by diabetes mellitus (DM), making it one of the most common serious medical complications of pregnancy [1-5]. The majority of cases $(>80 \%)$ are diagnosed for the first time during pregnancy (gestational DM); the remaining cases are pregestational DM (type 1 or type 2) [2]. Furthermore, the prevalence of diabetes in pregnancy is increasing in the United States, concurrent with the rising prevalence of obesity and type 2 diabetes in the general population; this increase is not explained by changes in the prevalence of other known maternal risk factors, such as advanced maternal age [6-8].

It is commonly stated that intrauterine exposure to maternal diabetes places offspring at increased risk for longterm adverse outcomes including overweight and obesity. In addition, it has been suggested that infants of women with diabetes should be specifically targeted for obesity prevention interventions. This suggestion raises several issues. Maternal obesity is also a risk factor for offspring overweight and obesity, is associated with maternal diabetes, and is a more prevalent condition than either gestational or pregestational DM [9-12]. It is unclear, therefore, whether it would be most effective to target infants of women with diabetes specifically, or all infants of women with prepregnancy obesity. Second, studies commonly cited as providing support for a causal association between maternal gestational DM and offspring overweight and obesity, including those conducted among Pima Indian women, have design limitations including combining pregestational and gestational DM into one exposure group, examination of exposed individuals only without a nondiabetic control group, and failure to control for important potential confounders [13-15].

In this study, we systematically reviewed studies examining the association between gestational DM and childhood overweight and obesity. We focused on gestational DM because it is the most prevalent form of diabetes in pregnancy. We summarized findings and addressed methodological limitations of previously published studies. We did not calculate a combined single estimate of the findings due to the heterogeneity of methodology and rigor among 
the studies. Finally, we provided recommendations regarding approaches for future studies.

\section{Materials and Methods}

2.1. Search Process. We used recommendations from the Meta-analysis of Observational Studies in Epidemiology (MOOSE) guidelines to identify studies for possible inclusion in this analysis [16]. We first searched PubMed records for the period January 1990-January 2011 using the following search terms for childhood overweight/obesity: (Pregn*) AND (GDM OR gestational diabetes OR diabetes OR glucose) AND (Overweight or obes* or BMI or body mass index or weight gain) AND (child* OR adolescen* OR offspring OR long term OR fetal program* OR imprint*). From this search, the full text was retrieved for papers in which the abstracts mentioned a relationship between maternal gestational DM and childhood overweight and obesity. Studies that did not have full text in English were translated for review.

Next, we manually reviewed the reference lists of the publications retrieved and obtained the entire text of publications that potentially could be included in the systematic review. Finally, we searched the reference lists of review articles on gestational DM and childhood overweight and obesity published in the last five years from January 2006 to January 2011 for additional potential publications. We did not attempt to locate any unpublished studies.

Studies that were considered potentially eligible were then reviewed for inclusion in the analysis based on the following criteria.

(1) Data were reported from a cohort with a nondiabetic exposure group or from a case-control study, and not from a case series.

(2) Cases of gestational DM were not combined with cases of pregestational DM. We did not exclude studies based on criteria for defining gestational DM.

(3) An association was reported (negative, positive, or null) for childhood (ages 2-18 years) overweight and/or obesity using a BMI-for-age-and-sex of $>85$ or $>95$ th percentile. We did not exclude studies based on the authors' reference population for child growth. However, we did exclude studies that examined BMI only as a continuous variable.

(4) The manuscript was written in English or in a language that could be translated with resources available at CDC.

(5) For studies with offspring outcome assessment at multiple ages, findings from data for longest duration of followup were used. When multiple articles from the same study population met the study criteria, we included only the publication with the most recent data available.

2.2. Data Abstraction. All articles were read and reviewed by two authors (S. Y. Kim and T. Njoroge). The authors abstracted the design, setting, location, and time period; the number and characteristics of study participants; the gestational DM diagnosis criteria; the source(s) for childhood obesity (e.g., medical records and clinical databases), and the statistical methods.

2.3. Statistical Methods. For each study, we constructed separate two-by-two tables to calculate unadjusted odds ratios (ORs) and 95\% confidence intervals (CIs) of gestational DM and each childhood weight outcome analyzed by the study's authors. If a study presented only prevalence estimates, we contacted the author to request the actual sample size for the percentages. We also presented the adjusted odds ratios when available.

\section{Results}

Childhood Obesity. We identified 1362 potentially relevant studies by searching PubMed; of these, 144 abstracts reported a finding on the relationship between maternal gestational DM and childhood obesity and the full texts of these articles were retrieved for detailed examination (Figure 1). We also reviewed the reference lists of all 144 studies, and identified an additional 48 studies for possible inclusion. After review of these 192 articles, 140 were excluded, either because they did not report the results of a cohort or case-control study or because they clearly did not address an association between maternal gestational DM and childhood obesity; thus, 52 studies were considered further for inclusion. Of these, 37 studies were excluded because gestational and pregestational DM were combined, there was no nondiabetic control group, or BMI was not presented as 85 th or 95 th percentile or it was presented only as a continuous variable. An additional 3 studies were excluded because later papers reported results on the same study population. A total of 12 studies met the inclusion criteria [17-28].

Six of the studies were conducted in the United States, and one each was conducted in Germany, Brazil, the United Kingdom, Hong Kong, Finland, or Poland (Table 1). We translated one study and contacted three authors. No studies were excluded because of lack of language translation resources. Sources of information on maternal gestational DM diagnosis included maternal self-report, clinical records, or blood glucose measurements. Information used to determine childhood obesity was obtained from parental report, anthropometric measurements obtained as part of a study protocol, hospital records, or clinical databases.

The crude odds ratios for the relationship between gestational DM and childhood overweight or obesity ranged from 0.7 to 6.3 (Figure 2(a)). When the studies were ordered from offspring overweight to obesity, the magnitude of the association did not increase with increasing levels of offspring BMI. If we exclude the lower and upper values, the OR ranges from 1.0 to 2.5. Eight of the twelve studies had significant findings in the crude analysis. In the three of the twelve studies (two of which were among the eight with a significant finding), the authors adjusted for potential confounders when examining the association between gestational DM and childhood obesity compared to a nondiabetic 


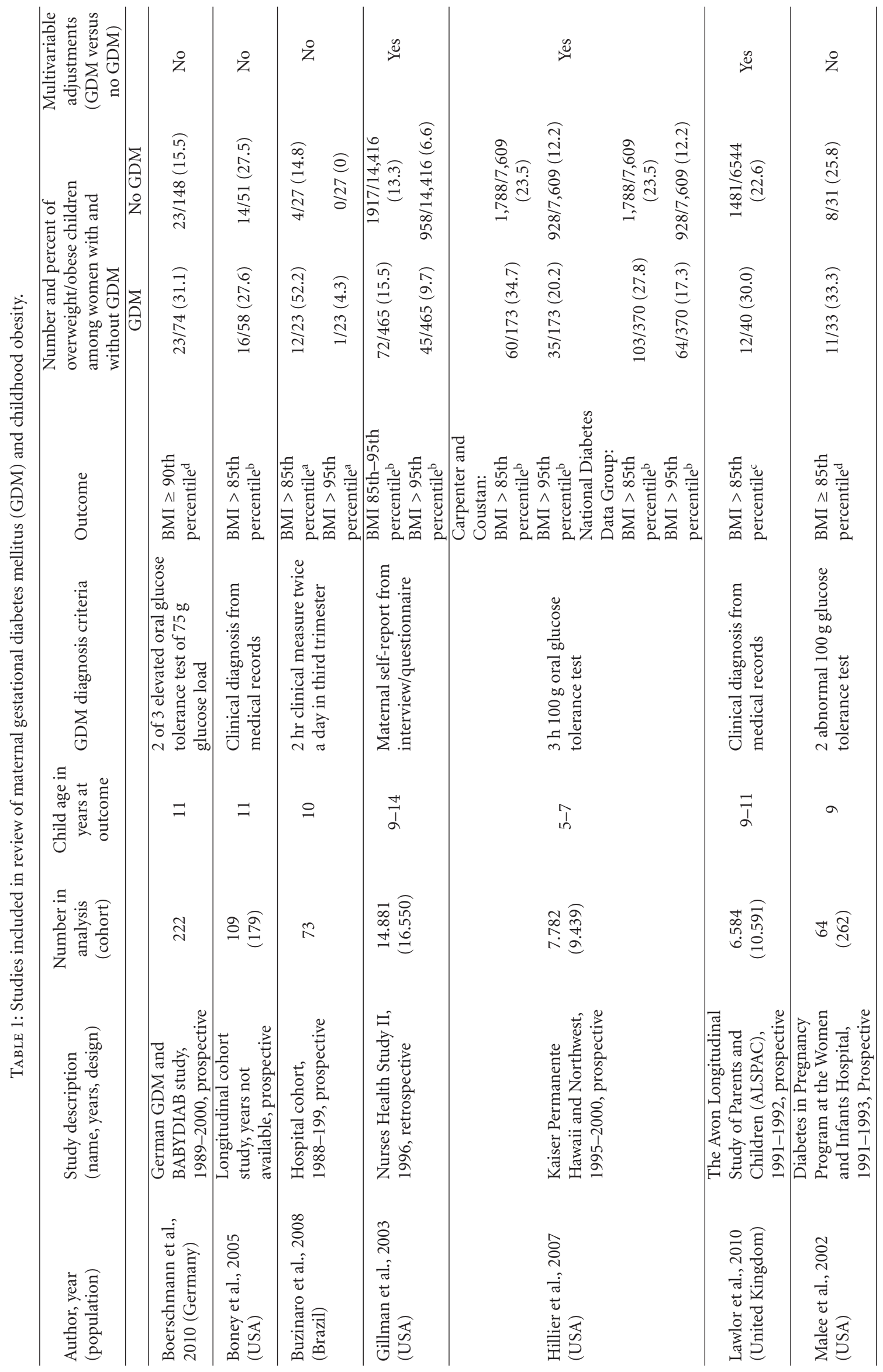




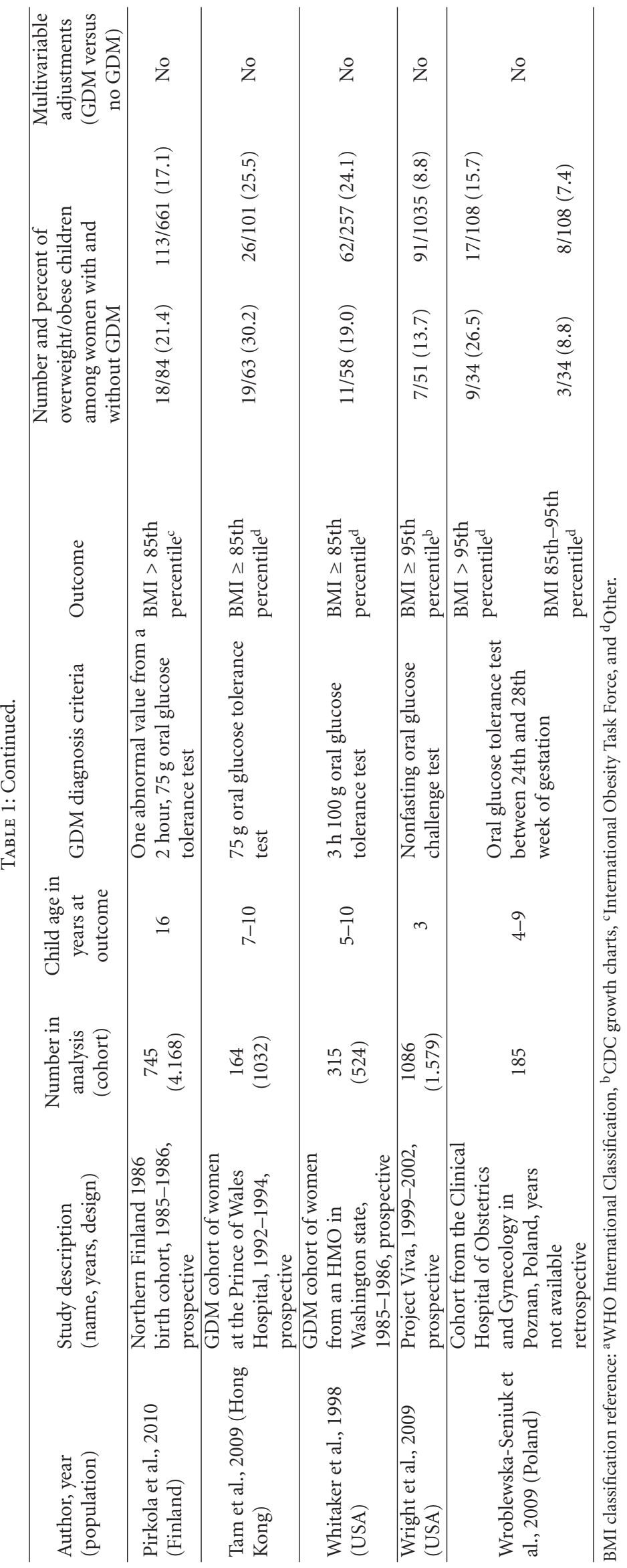




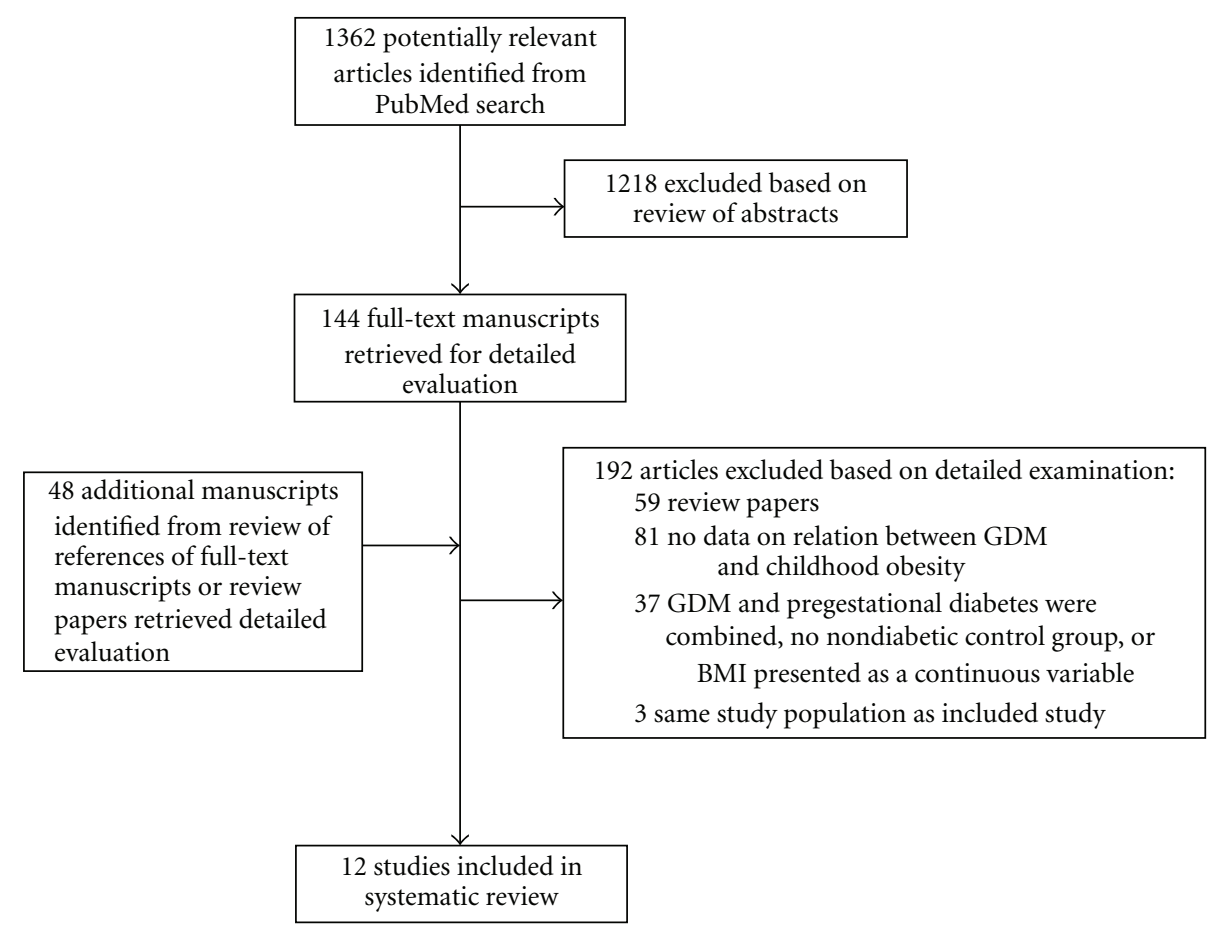

FIGURE 1: Flow diagram showing the number of studies included in and excluded from the systematic review for childhood obesity.

control group (Figure 2(b)). In the first of the three studies, Gillman and colleagues reported a crude odds ratio (OR) of $1.2(0.9-1.5)$ and $1.4(1.1-2.0)$ for adolescent weight at 85th-95th percentile and $>95$ th percentile, respectively at age 9-14 years old among the offspring of mothers with gestational DM versus no diabetes [19]. These estimates did not change after adjustment for level of child's physical activity, hours per week watching television, and energy intake. However, adjustment for maternal prepregnancy BMI attenuated the associations for both weight at 85th-95th percentile $(\mathrm{AOR}=1.0,95 \%$ CI $0.7-1.3)$ and at $>95$ th percentile $(\mathrm{AOR}=1.2,95 \% \mathrm{CI} 0.8-1.7)$, and results were no longer significant. In the second study, Lawlor and colleagues reported a crude OR of $1.5(0.8,2.9)$ for weight $>85$ th percentile at age 9-11 years among offspring of mothers with gestational DM, but this positive finding was reversed after adjustment for prepregnancy BMI (AOR = $0.62,95 \%$ CI $0.3,1.23$ ) [26]. However, the finding was not significant either before or after the adjustment. In the third study, by Hillier and colleagues, the authors compared treated and nontreated women with gestational DM each to women with no diabetes [20]. They did not adjust for prepregnancy BMI but did adjust for maternal age, parity, gestational weight gain, ethnicity, macrosomia, and sex of child. They reported an adjusted OR of $1.9(1.3,2.8)$ for child's weight $>85$ th percentile and $1.8(1.1,2.9)$ for child's weight $>95$ th percentile at age 5-7 years among offspring of mothers likely not treated for gestational DM (those who met criteria for gestational DM using Carpenter and Coustan criteria only and did not meet the National Diabetes Data Group (NDDG) criteria) compared to offspring of women with no diabetes. They found no significant associations between gestational diabetes and childhood weight $>85$ th percentile (adjusted OR $=1.3(0.8,1.9)$ or for child's weight $>95$ th percentile (adjusted $\mathrm{OR}=1.38(0.8,2.3)$ ) in women likely treated for gestational DM (those who met criteria for gestational DM using the NDDG criteria). In addition, the authors observed a dose-response relationship between maternal glucose concentration in quartiles among women with no diabetes and risk of childhood overweight and obesity, suggesting that elevated maternal blood glucose does affect risk of overweight and obesity in offspring, and that this risk may be reduced with treatment of gestational DM during pregnancy.

In one of the 12 studies, Boerschmann and colleagues did not adjust for any confounders; however, the authors did present results stratified by maternal BMI among offspring of women with gestational DM. They found that among offspring of women with gestational DM, there was an increase in offspring BMI percentile $\geq 90$ as maternal BMI increased. This increase further supports the strength of maternal BMI [27]. None of the 12 studies included information on genetic factors.

\section{Discussion}

To our knowledge, ours is the first study to systematically review all published case-control and cohort studies specifically examining the association between maternal gestational DM and the prevalence of offspring overweight and obesity. We excluded many studies from our review because of methodological limitations, including the use of an exposure group combining pregestational and gestational DM, and the lack of a nondiabetic control group. We found that 


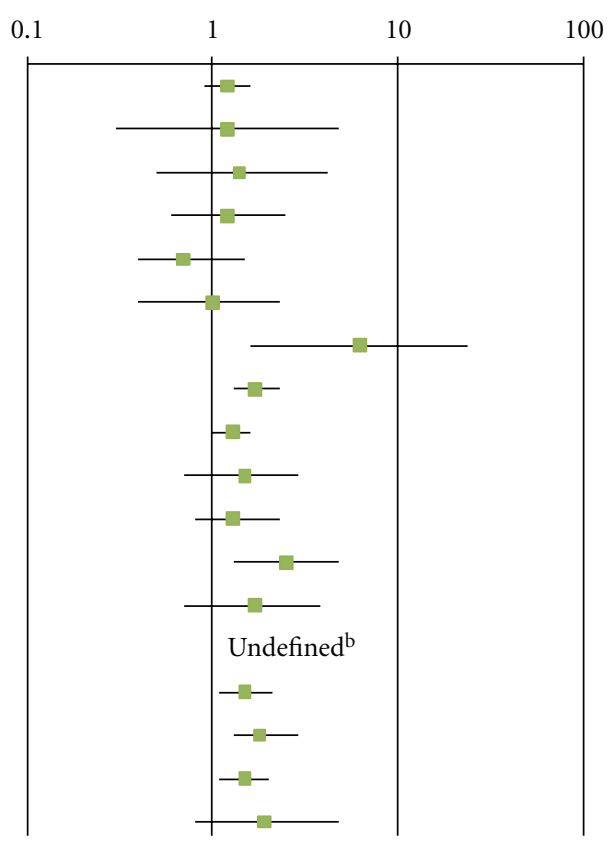

\begin{tabular}{|c|c|c|}
\hline Author (year) & $N$ & $\begin{array}{c}\text { Offspring } \\
\text { BMI percentile }\end{array}$ \\
\hline Gillman et al., (2003) & 14,881 & $85-95$ \\
\hline Wroblewska-Seniuk et al., (2009) & 185 & $85-95$ \\
\hline Malee et al., (2002) & 64 & $\geq 85$ \\
\hline Tam et al., (2009) & 164 & $\geq 85$ \\
\hline Whitaker et al., (1998) & 315 & $\geq 85$ \\
\hline Boney et al., (2005) & 109 & $>85$ \\
\hline Buzinaro et al., (2008) & 73 & $>85$ \\
\hline Hillier et al., (C and C; 2007) ${ }^{\mathrm{a}}$ & 7,782 & $>85$ \\
\hline Hillier et al., (NDDG; 2007) ${ }^{\mathrm{a}}$ & 7,782 & $>85$ \\
\hline Lawlor et al., (2010) & 6,584 & $>85$ \\
\hline Pirkola et al., (2010) & 745 & $>85$ \\
\hline Boerschmann et al., (2010) & 222 & $\geq 90$ \\
\hline Wright et al., (2009) & 1,086 & $\geq 95$ \\
\hline Buzinaro et al., $(2008)^{\mathrm{b}}$ & 73 & $>95$ \\
\hline Gillman et al., (2003) & 14,881 & $>95$ \\
\hline Hillier et al., (C and C; 2007) ${ }^{\mathrm{a}}$ & 7,782 & $>95$ \\
\hline Hillier et al., (NDDG; 2007) $)^{\mathrm{a}}$ & 7,782 & $>95$ \\
\hline Wroblewska-Seniuk et al., (2009) & 185 & $>95$ \\
\hline
\end{tabular}

(a)

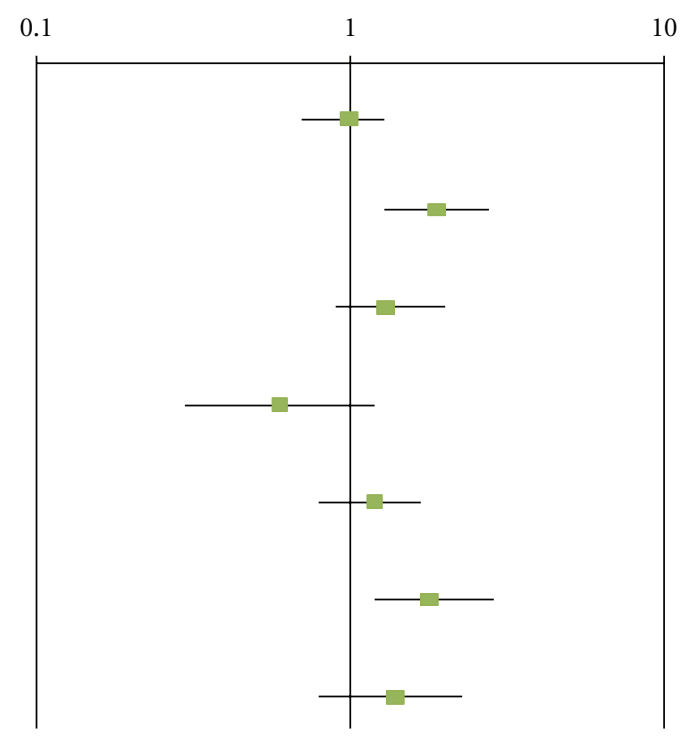

\begin{tabular}{lcc|}
\hline Author (year) & $N$ & $\begin{array}{c}\text { Offspring } \\
\text { BMI percentile }\end{array}$ \\
\hline Gillman et al., (2003) & 14,881 & $85-95$ \\
Hillier et al., (C and C; 2007) $)^{\mathrm{b}, \mathrm{d}}$ & 7,782 & $>85$ \\
Hillier et al., (NDDG; 2007) & 7,782 & $>85$ \\
Lawlor et al., (2010) & & $>85$ \\
Gillman et al., (2003) & 6,584 & $>95$ \\
Hillier et al., (C and C; 2007) & 7,782 & $>95$ \\
& & $>95$ \\
Hillier et al., (NDDG; 2007) & 7,782 & \\
\hline
\end{tabular}

(b)

FIgURe 2: (a) Association of GDM and childhood overweight or obesity, unadjusted odds ratio, and 95\% confidence interval.

${ }^{a}$ At the time this analysis was conducted, Kaiser Permanente used the NDDG criteria to diagnose and treat GDM. However, in the analysis, they calculated GDM using both criteria. Therefore, those meeting the NDDG criteria in this analysis were likely treated with diet or diet/insulin, but those meeting only the Carter and Coustan criteria were likely to not be treated. ${ }^{\text {b}}$ Undefined because odds ratio could not be calculated with a zero cell. (b) Association of GDM and childhood overweight or obesity compared to a nondiabetic control group among studies that adjusted for any confounders, adjusted odds ratios, and 95\% confidence intervals. adjusted for maternal BMI and child's age, gender, Tanner stage, TV watching, physical activity, energy intake, birth weight, breastfeeding duration, birth order, and mom's household income, mother's smoking, dietary restraint, weight cycling, weight concerns, and mother's current BMI. ' Adjusted for maternal age, parity, weight gain during pregnancy, ethnicity, macrosomia at birth, and sex of child. 'Adjusted for maternal prepregnancy BMI and sex, age at outcome, height, height squared, maternal age, social class, parity, smoking during pregnancy, mode of delivery, and maternal prepregnancy BMI. ${ }^{\mathrm{d}}$ At the time this analysis was conducted, Kaiser Permanente used the NDDG criteria to diagnose and treat GDM. However, in the analysis, they calculated GDM using both criteria. Therefore, those meeting the NDDG criteria in this analysis were likely treated with diet or diet/insulin, but those meeting only the Carter and Coustan criteria were likely to not be treated. 
studies of the effects of maternal gestational DM on offspring overweight and obesity have yielded inconclusive results, which is consistent with a recent review examining maternal diabetes and offspring BMI $\mathrm{z}$-scores, where they found the association between maternal diabetes and offspring BMI to be no longer significant after adjustment for prepregnancy BMI [29].

\section{Fetal Exposure to Maternal Hyperglycemia}

Women with preexisting diabetes have a substantially increased risk of pregnancy complications including fetal loss, perinatal mortality, and of delivering an infant with congenital anomalies [30]. Although the mechanisms underlying these associations are not completely understood, it has been shown that tight glycemic control in early pregnancy reduces the prevalence of such pregnancy complications [31]. In contrast to women with pregestational DM who are hyperglycemic throughout pregnancy, women with gestational DM typically develop hyperglycemia in the second or third trimester of pregnancy, and pregnancy complications associated with gestational DM are not as severe [32, 33]. Therefore, we cannot assume that pregestational DM and gestational DM have the same effects on fetal development and long-term offspring outcome, and it is important to study associations between these two conditions and offspring outcomes separately.

In addition to timing of exposure, it is important to consider differences in diagnostic cutpoints for gestational DM. Depending on the cutpoints, it may result in the inclusion of women with milder disease in the gestational DM exposed group, which could attenuate the association between gestational DM and offspring overweight and obesity. Therefore, the severity of disease would be important to consider when examining offspring overweight and obesity.

\section{Confounders}

6.1. Maternal Prepregnancy Obesity. Studying the relationships between pregestational and gestational DM and risk of overweight and obesity in the offspring is complicated by difficulties in fully controlling for potential confounders, including maternal obesity, genetic factors, and maternal and infant lifestyle. In our review, we found that while many authors reported positive associations between gestational DM and childhood obesity in crude analysis, their results were not adjusted for important potential confounders, most notably maternal obesity. In the three studies that included adjusted analysis, the associations were attenuated after adjustment for confounders. Furthermore, results were adjusted for maternal obesity in only two studies; in both, associations between gestational DM and childhood overweight or obesity were not significant after adjustment.

Maternal obesity is an important risk factor for gestational DM; women who are overweight, obese, or severely obese before pregnancy are two-, four-, and eight-times more likely to develop gestational DM compared with normalweight women [11]. Infants born to overweight and obese mothers are more likely to be macrosomic, even in the absence of gestational diabetes [34]. In a recent study by Catalano et al., the authors found that prepregnancy obesity was significantly associated with offspring being in the highest BMI tertile and for having metabolic dysregulation at age 6-11 years [35]. They also found that prepregnancy obesity was the strongest perinatal predictor of high BMI in childhood, stronger than either maternal glucose homeostasis or weight gain during pregnancy. Although this study was not included in our review because the outcomes were not defined as overweight or obesity, the results provide additional support that gestational DM cannot be evaluated as an independent risk factor for childhood overweight and obesity without accounting for potential confounding from maternal obesity. This concept is further supported by a follow-up study of offspring of women who participated in the Hyperglycemia and Adverse Pregnancy Outcome Study in Belfast, Northern Ireland. The authors found that at age two the overall correlations between maternal glucose during pregnancy and BMI z-score were weak and that birth weight and maternal BMI remained independent predictors of BMI z-score after adjustment [36].

6.2. Other Potential Confounders. Other potentially important confounders in the association between gestational DM and offspring overweight and obesity include common social, environmental, and genetic factors shared by both the mothers and the offspring. Poor dietary habits and a sedentary lifestyle in the mother may contribute to increased prepregnancy weight and weight gain during pregnancy, and may increase the risk of poor diet and sedentary behavior for the offspring [10]. However, only one of the studies in our review examined the association between physical activity and nutrition and childhood overweight and obesity [19]. Genetic influence is also important to consider. However, it is difficult to fully control for genetics outside of sibling studies, and none of the studies in our review included genetic factors as potential confounders. To our knowledge, no studies examining associations between gestational DM and offspring obesity have taken all of potentially important confounders, including maternal obesity, gestational weight gain, and maternal and infant lifestyle and genetic factors into account.

\section{Treatment of Gestational DM}

If treatment of gestational DM reduces risk of overweight or obesity in offspring, it may be difficult to consistently detect an association in observational studies. Women with gestational DM are typically instructed to monitor blood sugar and adjust their dietary habits, which may improve longterm offspring outcomes. In only one study did the authors examine treated and nontreated women with gestational DM [20]. They reported significant associations with childhood overweight and with obesity in the nontreated group but not in the treated group. Further evidence that treatment of gestational DM may improve offspring outcome is found in studies of women with high blood glucose concentration, 
but not sufficiently high for a diagnosis of gestational DM thus not treated. For example, Deierlein et al. found that fetal exposure to maternal glucose concentration in the high-normal range was associated with the development of overweight and obesity in the offspring at age three years, independent of maternal prepregnancy BMI [37].

\section{Future Studies}

As previously discussed, most existing observational studies cannot be used to definitively quantify the independent contribution of gestational diabetes to offspring overweight and obesity risk because of unmeasured confounding and other methodological limitations. We recommend the following for future studies.

(1) Observational studies should analyze women with pregestational and gestational DM separately, and if possible include documentation of timing of fetal exposure to elevated maternal glucose levels.

(2) Observational studies should include documentation of lifestyle and other environmental factors present during infancy and childhood.

(3) All studies should address relevant confounders, including maternal prepregnancy BMI. One possible approach would be to make use of databases with linked siblings so outcomes between siblings with discordant exposure to gestational DM could be compared, providing a mechanism to control for shared genetic and lifestyle factors.

(4) When possible, randomized trials for the treatment for mild gestational DM should include long-term followup of offspring assess the effects of maternal interventions during pregnancy on infant and child outcomes [38]. If it can be confirmed that treatment of gestational DM is associated with a reduced risk of overweight or obesity in offspring, it will provide further evidence that there is a causal relationship and that an effective prevention intervention exists.

\section{Limitations}

This study has some limitations. First, we did not include unpublished studies, therefore our results may be affected by publication bias. In addition, we may have missed studies that were not listed in PubMed or referenced in other published studies or reviews. Finally, these studies are not all directly comparable due to discrepancies in the study population, methodology, gestational DM diagnostic criteria, BMI reference population, and ages. However, the present study is the first to systematically review all published case-control and cohort studies examining the association between maternal gestational DM and the prevalence of offspring overweight and obesity.

\section{Conclusions}

In conclusion, this review demonstrates that studies of associations between maternal gestational DM on offspring overweight and obesity have yielded inconclusive results. Because maternal obesity is a more prevalent condition than gestational DM and is strongly associated with offspring obesity, we need a better understanding of the relative contributions of maternal obesity and gestational DM to risk before designating infants of women with GDM specifically as targets for obesity prevention efforts. Interventions addressing prepregnancy obesity may have a greater public health impact on childhood overweight and obesity than those targeting offspring of women with gestational DM. A stronger body of evidence is needed to better understand the potential associations between maternal gestational DM and offspring outcomes.

\section{Disclosure}

The findings and conclusions in this report are those of the authors and do not necessarily represent the official position of the Centers for Disease Control and Prevention.

\section{Acknowledgment}

The authors would like to thank Connie Bish and Susan Chu for their feedback and contributions at the inception of this paper.

\section{References}

[1] F. Cunningham, N. Gant, K. Leveno, L. Gilstrap, J. Hauth, and K. Wenstrom, Williams Obstetrics, McGrow-Hill, New York, NY, USA, 21st edition, 2001.

[2] S. S. Albrecht, E. V. Kuklina, P. Bansil et al., "Diabetes trends among delivery hospitalizations in the U.S., 19942004," Diabetes Care, vol. 33, no. 4, pp. 768-773, 2010.

[3] D. Getahun, C. Nath, C. V. Ananth, M. R. Chavez, and J. C. Smulian, "Gestational diabetes in the United States: temporal trends 1989 through 2004," American Journal of Obstetrics and Gynecology, vol. 198, no. 5, pp. 525.e1-525.e5, 2008.

[4] S. Y. Chu, K. Abe, L. R. Hall, S. Y. Kim, T. Njoroge, and C. Qin, "Gestational diabetes mellitus: all Asians are not alike," Preventive Medicine, vol. 49, no. 2-3, pp. 265-268, 2009.

[5] S. Y. Kim, L. England, H. G. Wilson, C. Bish, G. A. Satten, and P. Dietz, "Percentage of gestational diabetes mellitus attributable to overweight and obesity," American Journal of Public Health, vol. 100, no. 6, pp. 1047-1052, 2010.

[6] A. H. Mokdad, E. S. Ford, B. A. Bowman et al., "Prevalence of obesity, diabetes, and obesity-related health risk factors, 2001," Journal of the American Medical Association, vol. 289, no. 1, pp. 76-79, 2003.

[7] S. Y. Kim, P. M. Dietz, L. England, B. Morrow, and W. M. Callaghan, "Trends in pre-pregnancy obesity in nine states, 1993-2003," Obesity, vol. 15, no. 4, pp. 986-993, 2007.

[8] D. Dabelea, J. K. Snell-Bergeon, C. L. Hartsfield, K. J. Bischoff, R. F. Hamman, and R. S. McDuffie, "Increasing prevalence of gestational diabetes mellitus (GDM) over time and by birth cohort: Kaiser Permanente of Colorado GDM screening program," Diabetes Care, vol. 28, no. 3, pp. 579-584, 2005.

[9] V. Svensson, J. A. Jacobsson, R. Fredriksson et al., "Associations between severity of obesity in childhood and adolescence, obesity onset and parental BMI: a longitudinal cohort study," International Journal of Obesity, vol. 35, pp. 46-52, 2011. 
[10] V. Burke, L. J. Beilin, and D. Dunbar, "Family lifestyle and parental body mass index as predictors of body mass index in Australian children: a longitudinal study," International Journal of Obesity, vol. 25, no. 2, pp. 147-157, 2001.

[11] S. Y. Chu, W. M. Callaghan, S. Y. Kim et al., "Maternal obesity and risk of gestational diabetes mellitus," Diabetes Care, vol. 30, no. 8, pp. 2070-2076, 2007.

[12] S. Y. Chu, S. Y. Kim, and C. L. Bish, "Prepregnancy obesity prevalence in the United States, 2004-2005," Maternal and Child Health Journal, vol. 13, no. 5, pp. 614-620, 2009.

[13] D. Dabelea, "The predisposition to obesity and diabetes in offspring of diabetic mothers," Diabetes Care, vol. 30, no. 2, pp. S169-S174, 2007.

[14] B. L. Silverman, L. Landsberg, and B. E. Metzger, "Fetal hyperinsulinism in offspring of diabetic mothers. Association with the subsequent development of childhood obesity," Annals of the New York Academy of Sciences, vol. 699, pp. 3645, 1993.

[15] B. E. Metzger, "Long-term outcomes in mothers diagnosed with gestational diabetes mellitus and their offspring," Clinical Obstetrics and Gynecology, vol. 50, no. 4, pp. 972-979, 2007.

[16] D. F. Stroup, J. A. Berlin, S. C. Morton et al., "Meta-analysis of observational studies in epidemiology: a proposal for reporting," Journal of the American Medical Association, vol. 283, no. 15, pp. 2008-2012, 2000.

[17] C. M. Boney, A. Verma, R. Tucker, and B. R. Vohr, "Metabolic syndrome in childhood: association with birth weight, maternal obesity, and gestational diabetes mellitus," Pediatrics, vol. 115, no. 3, pp. e290-e296, 2005.

[18] E. F. Buzinaro, C. B. Berchieri, A. L. M. Haddad, C. R. Padovani, and W. De Paula Pimenta, "Overweight in adolescent offspring of women with hyperglycemia during pregnancy," Arquivos Brasileiros de Endocrinologia e Metabologia, vol. 52, no. 1, pp. 85-92, 2008.

[19] M. W. Gillman, S. Rifas-Shiman, C. S. Berkey, A. E. Field, and G. A. Colditz, "Maternal gestational diabetes, birth weight, and adolescent obesity," Pediatrics, vol. 111, no. 3, pp. e221e226, 2003.

[20] T. A. Hillier, K. L. Pedula, M. M. Schmidt, J. A. Mullen, M. A. Charles, and D. J. Pettitt, "Childhood obesity and metabolic imprinting: the ongoing effects of maternal hyperglycemia," Diabetes Care, vol. 30, no. 9, pp. 2287-2292, 2007.

[21] M. P. Malee, A. Verma, G. Messerlian, R. Tucker, and B. R. Vohr, "Association between maternal and child leptin levels 9 years after pregnancy complicated by gestational diabetes," Hormone and Metabolic Research, vol. 34, no. 4, pp. 212-216, 2002.

[22] R. C. Whitaker, M. S. Pepe, K. D. Seidel, J. A. Wright, and R. H. Knopp, "Gestational diabetes and the risk of offspring obesity," Pediatrics, vol. 101, no. 2, p. E9, 1998.

[23] W. H. Tam, R. C. W. Ma, X. Yang et al., "Glucose intolerance and cardiometabolic risk in children exposed to maternal gestational diabetes mellitus in utero," Pediatrics, vol. 122, no. 6, pp. 1229-1234, 2008.

[24] C. S. Wright, S. L. Rifas-Shiman, J. W. Rich-Edwards, E. M. Taveras, M. W. Gillman, and E. Oken, "Intrauterine exposure to gestational diabetes, child adiposity, and blood pressure," American Journal of Hypertension, vol. 22, no. 2, pp. 215-220, 2009.

[25] K. Wroblewska-Seniuk, E. Wender-Ozegowska, and J. Szczapa, "Long-term effects of diabetes during pregnancy on the offspring," Pediatric Diabetes, vol. 10, no. 7, pp. 432-440, 2009.

[26] D. A. Lawlor, A. Fraser, R. S. Lindsay et al., "Association of existing diabetes, gestational diabetes and glycosuria in pregnancy with macrosomia and offspring body mass index, waist and fat mass in later childhood: findings from a prospective pregnancy cohort," Diabetologia, vol. 53, no. 1, pp. 89-97, 2010.

[27] H. Boerschmann, M. Pflüger, L. Henneberger, A. G. Ziegler, and S. Hummel, "Prevalence and predictors of overweight and insulin resistance in offspring of mothers with gestational diabetes mellitus," Diabetes Care, vol. 33, no. 8, pp. 1845-1849, 2010.

[28] J. Pirkola, A. Pouta, A. Bloigu et al., "Risks of overweight and abdominal obesity at age 16 years associated with prenatal exposures to maternal prepregnancy overweight and gestational diabetes mellitus," Diabetes Care, vol. 33, no. 5, pp. 1115-1121, 2010.

[29] L. H. Philipps, S. Santhakumaran, C. Gale et al., "The diabetic pregnancy and offspring BMI in childhood: a systematic review and meta-analysis," Diabetologia, vol. 54, no. 8, pp. 1957-1966, 2011.

[30] F. Dunne, P. Brydon, K. Smith, and H. Gee, "Pregnancy in women with Type 2 diabetes: 12 years outcome data 19902002," Diabetic Medicine, vol. 20, no. 9, pp. 734-738, 2003.

[31] J. L. Kitzmiller, J. M. Block, F. M. Brown et al., "Managing preexisting diabetes for pregnancy: summary of evidence and consensus recommendations for care," Diabetes Care, vol. 31, no. 5, pp. 1060-1079, 2008.

[32] B. M. Casey, M. J. Lucas, D. D. McIntire, and K. J. Leveno, "Pregnancy outcomes in women with gestational diabetes compared with the general obstetric population," Obstetrics and Gynecology, vol. 90, no. 6, pp. 869-873, 1997.

[33] X. Xiong, L. D. Saunders, F. L. Wang, and N. N. Demianczuk, "Gestational diabetes mellitus: prevalence, risk factors, maternal and infant outcomes," International Journal of Gynecology and Obstetrics, vol. 75, no. 3, pp. 221-228, 2001.

[34] P. M. Catalano and H. M. Ehrenberg, "The short- and longterm implications of maternal obesity on the mother and her offspring," BJOG, vol. 113, no. 10, pp. 1126-1133, 2006.

[35] P. M. Catalano, K. Farrell, A. Thomas et al., "Perinatal risk factors for childhood obesity and metabolic dysregulation," American Journal of Clinical Nutrition, vol. 90, no. 5, pp. 13031313, 2009.

[36] D. J. Pettitt, S. McKenna, C. McLaughlin, C. C. Patterson, D. R. Hadden, and D. R. McCance, "Maternal glucose at 28 weeks of gestation is not associated with obesity in 2-year-old offspring: the Belfast Hyperglycemia and Adverse Pregnancy Outcome (HAPO) family study," Diabetes Care, vol. 33, no. 6, pp. 12191223, 2010.

[37] A. L. Deierlein, A. M. Siega-Riz, K. Chantala, and A. H. Herring, "The association between maternal glucose concentration and child BMI at age 3 years," Diabetes Care, vol. 34, no. 2, pp. 480-484, 2011.

[38] M. B. Landon, C. Y. Spong, E. Thom et al., "A multicenter, randomized trial of treatment for mild gestational diabetes," New England Journal of Medicine, vol. 361, no. 14, pp. 13391348, 2009. 


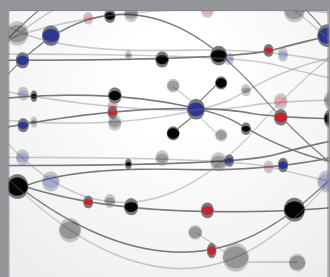

The Scientific World Journal
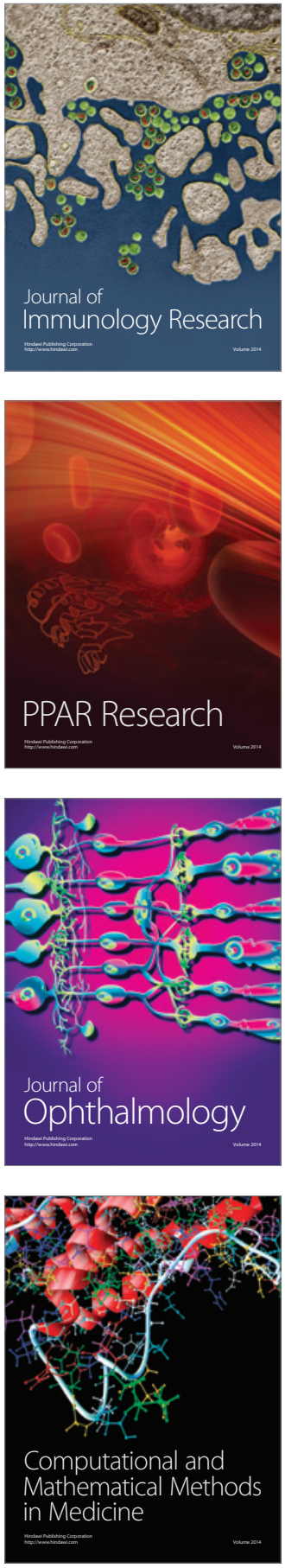

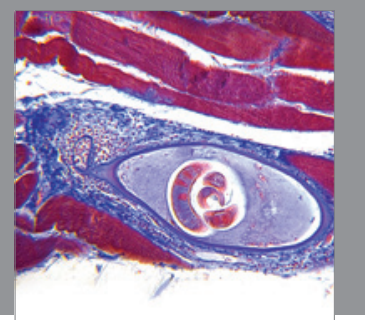

Gastroenterology

Research and Practice
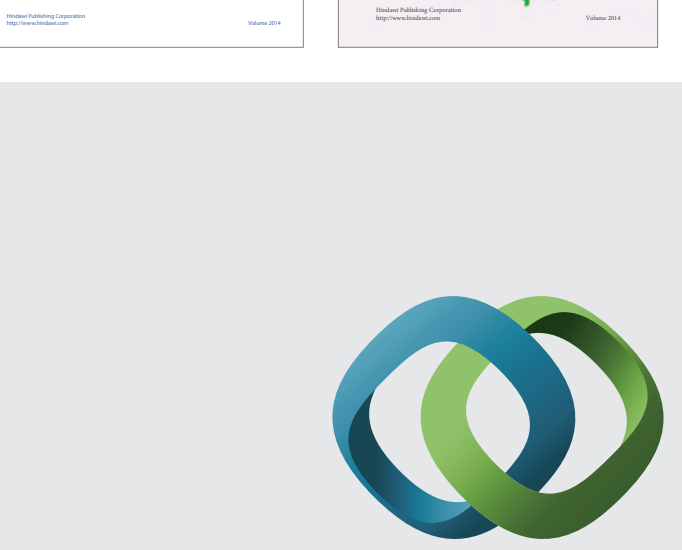

\section{Hindawi}

Submit your manuscripts at

http://www.hindawi.com
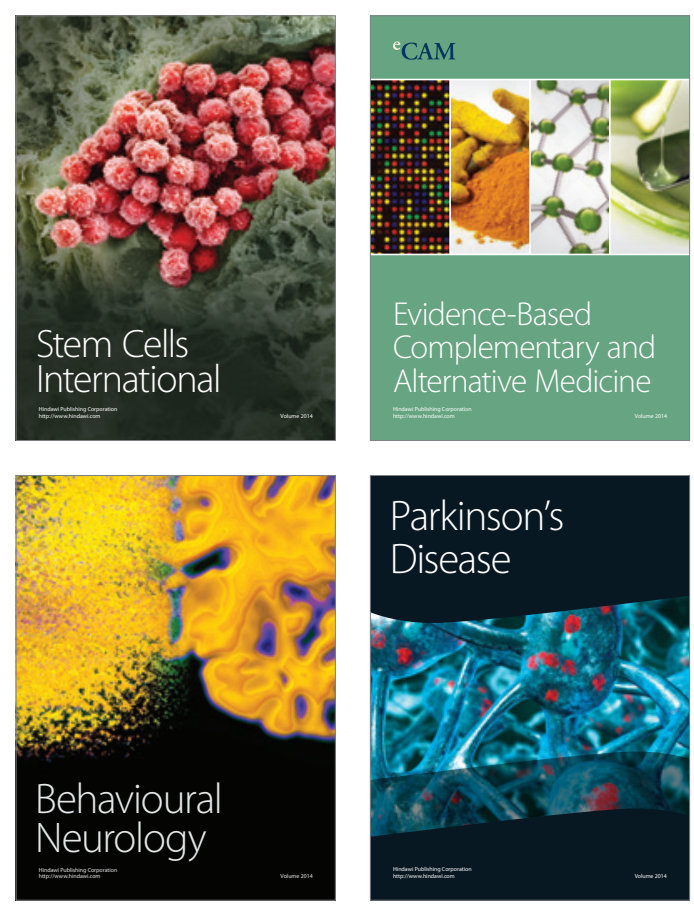

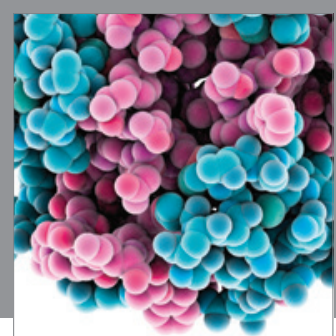

Journal of
Diabetes Research

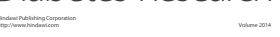

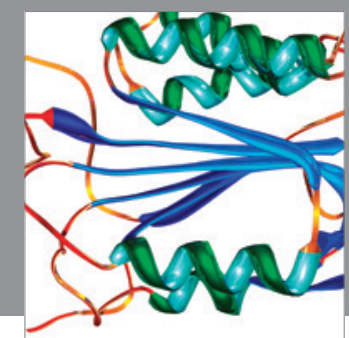

Disease Markers
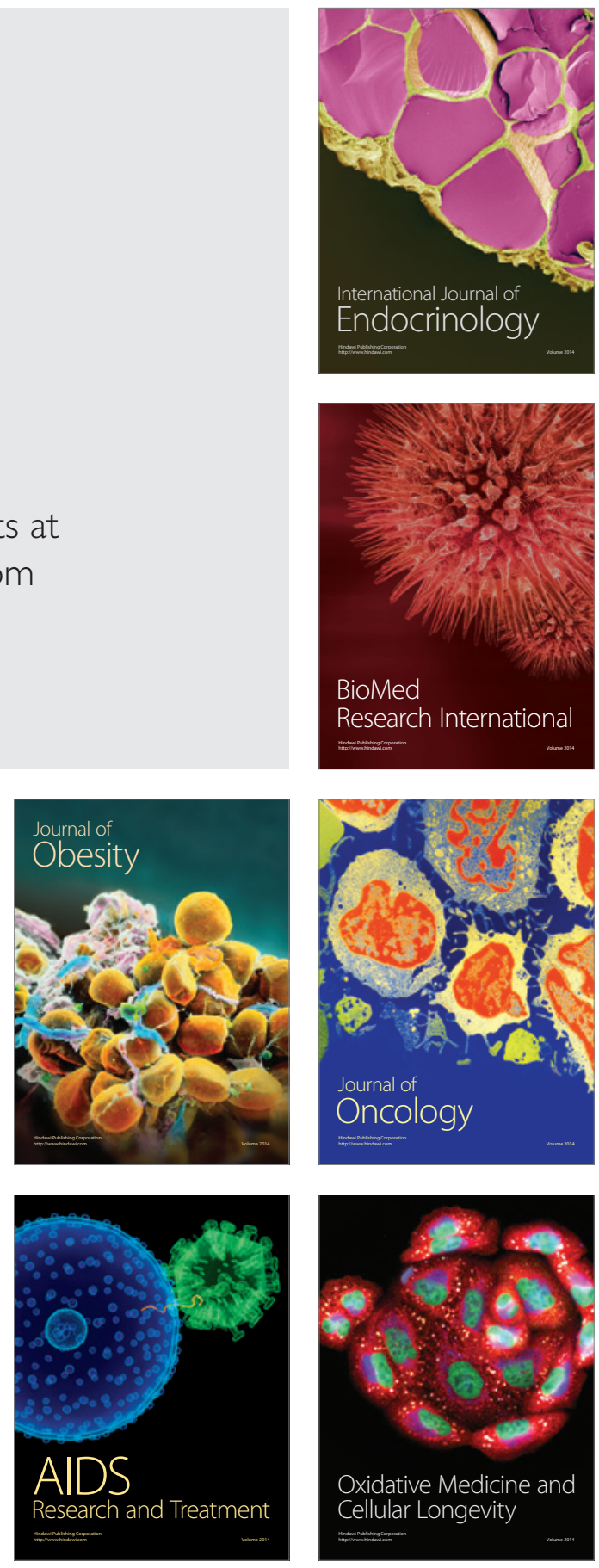